\title{
Cognitive behavioural therapy improved most sleep outcomes in insomnia secondary to chronic pain
}

Currie SR, Wilson KG, Pontefract AJ, et al. Cognitive-behavioral treatment of insomnia secondary to chronic pain.

J Consult Clin Psychol 2000 Jun;68:407-16.

\section{QUESTION: In patients with insomnia secondary to chronic pain, does cognitive behavioural therapy (CBT) improve sleep outcomes?}

\section{Design}

Randomised \{allocation concealed*\}†, unblinded*, controlled trial with 3 months of follow up.

\section{Setting}

3 clinics in Canada.

\section{Patients}

60 patients who were $<60$ years of age (mean age $45 \mathrm{y}$, $55 \%$ women), had non-malignant chronic pain of a musculoskeletal origin but not fibromyalgia (mean duration $9.2 \mathrm{y}$ ), reported sleep difficulties (mean duration of insomnia $7.9 \mathrm{y}$ ), and had no major medical or psychiatric disorder. Primary pain sites were back $(72 \%)$, neck $(20 \%)$, lower limbs $(5 \%)$, or pelvic region $(3 \%) .67 \%$ of patients used medication regularly. Follow up was $85 \%$.

\section{Intervention}

Patients were allocated to CBT $(n=32)$ or to a waiting list/self monitoring group $(n=28)$ for 7 weeks. CBT consisted of 7 two hour weekly sessions with groups of 5-7 participants. Each session involved a review of sleep diaries and progress, education about sleep and the causes of chronic insomnia, behaviour therapy targeting problems with sleep onset and maintenance, relaxation training, cognitive restructuring procedures, and sleep hygiene education. Patients in the CBT group were also given a patient oriented manual.

\section{Main outcome measures}

Sleep quantity and quality (sleep diaries and the Pittsburgh Sleep Quality Index) and nocturnal activity levels (actigraph monitors). Secondary outcomes were pain severity (Multidimensional Pain Inventory Pain Severity scale), emotional distress (Beck Depression Inventory), and medication use (Medication Quantification Scale).

\section{Main results}

Analysis was by intention to treat. CBT was better than self monitoring for improving sleep onset latency $(\mathrm{p}<0.025)$, sleep efficiency $(\mathrm{p}<0.001)$, wake time after sleep onset $(\mathrm{p}<0.005)$, and sleep quality $(\mathrm{p}<0.001)$ at 3 months (table). Level of nocturnal activity was more

Cognitive behavioural therapy $(C B T)$ v waiting list/self monitoring (control) for insomnia secondary to chronic pain

\begin{tabular}{lccc} 
& \multicolumn{2}{c}{ Mean } & \\
\cline { 2 - 3 } Outcomes at 3 months & CBT & Control & Mean difference (95\% CI) \\
Sleep onset latency $(\mathrm{min})$ & 27.8 & 46.8 & $19.0(4.1$ to 33.9$)$ \\
\hline Sleep efficiency & $84 \%$ & $71 \%$ & $13 \%(6$ to 20$)$ \\
\hline Wake after sleep onset $(\mathrm{min})$ & 51.6 & 97.5 & $45.9(17.4$ to 74.4$)$ \\
\hline PSQI score & 7.9 & 13.5 & $5.6(3.7$ to 7.5$)$ \\
\hline
\end{tabular}

‡PSQI=Pittsburgh Sleep Quality Index. Mean difference and $\mathrm{Cl}$ calculated from data in article.

improved in the CBT group than in the control group at 7 weeks (mean difference 5.1 per $15 \mathrm{sec}, 95 \%$ CI 2.1 to 8.1 ) but not at 3 months. Groups did not differ for awakenings, total sleep time, pain severity, emotional distress, or medication use.

\section{Conclusion}

In people with insomnia secondary to chronic pain, cognitive behavioural therapy improved most sleep outcomes.

*See glossary.

$\dagger$ Information provided by author.

\section{COMMENTARY}

The quality of life of patients with a chronic medical condition is also determined by the daily consequences of their frequently existing chronic sleep complaints. Surprisingly, the treatment of chronic insomnia in such patients is a neglected area of research. Although pharmacotherapy is the most widely used treatment of acute insomnia, its efficacy in the long term treatment of chronic insomnia remains unclear. ${ }^{1}$ According to Kripke, use of hypnotic medication becomes chronic because of the patient's incorrect attributions of benefit concerning sleep duration and daytime functioning. ${ }^{2}$

Currie et al have published a timely study in the non-pharmacological treatment of insomnia in patients with chronic pain. They showed that their CBT approachwhich is a "package" with different treatment modalitieswas more effective after 3 months than a self monitoring/ waiting list condition.

Although the study is a welcome contribution to the clinical treatment possibilities in such patients, a few remarks for daily practice have to be made. CBT includes techniques which enhance the ability to cope with stresses that may be contributing to insomnia, and aims to change assumptions and perceptions about insomnia. It is important to identify the main problems with sleep and to modify both thoughts and behaviour in certain patients. CBT requires a good relationship between the patient and the therapist and is most effective if the patient has an active coping style. ${ }^{3}$ Not all kinds of insomnia can be treated with CBT, such as insomnia resulting from (severe) depression, sleep apnoea, or periodic limb movements in sleep. Therefore, patients with a chronic medical condition should also have a careful, and if necessary repeated, diagnostic evaluation of their sleep complaints.

Alex L van Bemmel, MD, PhD Maastricht University Maastricht, the Netherlands

1 Lamberg L. Sleep specialists weigh hypnotics, behavioral therapies for insomnia. JAMA 1997;278:1647-9.

2 Kripke DF. Chronic hypnotic use: deadly risks, doubtful benefit. Sleep Medicine Reviews 2000;4:5-20.

3 Shneerson JM. Handbook of sleep medicine. Oxford: Blackwell Science, 2000. 